\title{
Article
}

\section{Melting and Crystallization of Differently Oriented Sets of Crystallites in Hard-Elastic Polypropylene}

N. Stribeck, U. No\#chel, and S. S. Funari

Macromolecules, 2009, 42 (6), 2093-2101• DOI: 10.1021/ma8025282 • Publication Date (Web): 24 February 2009

Downloaded from http://pubs.acs.org on March 18, 2009

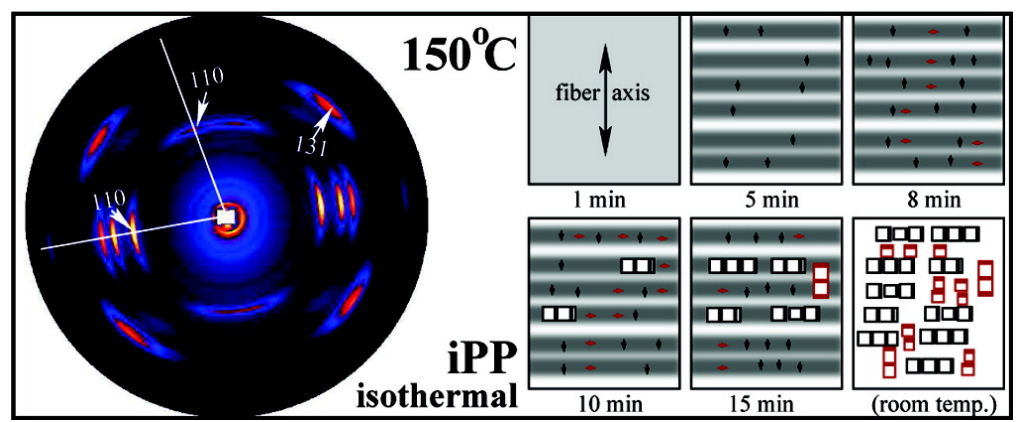

\section{More About This Article}

Additional resources and features associated with this article are available within the HTML version:

- $\quad$ Supporting Information

- Access to high resolution figures

- $\quad$ Links to articles and content related to this article

- Copyright permission to reproduce figures and/or text from this article

\section{View the Full Text HTML}




\title{
Melting and Crystallization of Differently Oriented Sets of Crystallites in Hard-Elastic Polypropylene
}

\author{
N. Stribeck, ${ }^{*}{ }^{\dagger}$ U. Nöchel, ${ }^{\dagger}$ and S. S. Funari ${ }^{\ddagger}$ \\ Institute of Technical and Macromolecular Chemistry, University of Hamburg, Bundesstr. 45, \\ 20146 Hamburg, Germany, and HASYLAB at DESY, Notkestr. 85, 22603 Hamburg, Germany
}

Received November 11, 2008; Revised Manuscript Received January 19, 2009

\begin{abstract}
Uniaxially oriented polypropylene (PP) is molten and crystallized isothermally from the oriented, quiescent melt. Nucleation and growth of differently oriented sets of crystallites ( $c$ set and $a^{*}$ set) appear to be decoupled. After shallow quench, crystallization is preceded by (spinodal) decomposition. Evolution is monitored by wide-angle X-ray scattering (WAXS) and compared with results of a SAXS study. Peak integrals (crystallinity) and minimum crystallite size are tracked. In the commercial starting material, $a^{*}$-set crystallites melt at $158{ }^{\circ} \mathrm{C}$. The $c$ set melts at $170{ }^{\circ} \mathrm{C}$ furnace temperature. After recrystallization, both sets melt at $170{ }^{\circ} \mathrm{C}$. Isothermal crystallization is divided into two distinct phases. During nucleation, the crystallinity stays low. The second phase is dominated by crystallinity growth. At $150{ }^{\circ} \mathrm{C}$, the $c$ set is seeded first. At 145 and $140{ }^{\circ} \mathrm{C} a^{*}$-oriented crystallites are the first. The first-seeded set starts to grow first. $c$-set crystallinity is always growing faster than $a^{*}$-set crystallinity. The evolution of the SAXS cross-diagram in the growth phase can be explained by both lamellae growing at right angles and block merging.
\end{abstract}

\section{Introduction}

It is well known ${ }^{1}$ that uniaxially oriented polypropylene (PP) exhibits a bimodal crystallite orientation. In one of the two sets, the unit-cell $c$ axis is parallel to the fiber axis. In the other set, the $a^{*}$ axis is in the fiber direction. The $a^{*}$ direction $^{2}$ is the normal on the $b c$ plane of the unit cell. During melt-spinning of PP, first, the wide-angle X-ray scattering (WAXS) reflections of the $c$-set crystallites appear ${ }^{3}$ at the equator. Later, the quasimeridional reflections of the $a^{*}$ set show up. In the electron microscope, a corresponding bimodal nanostructure has been discovered, the so-called cross-hatched structure. ${ }^{4}$ It contains a second set of lamellae that are oriented transverse to the normal layers. They contain $a^{*}$-oriented crystallites and are considered to be formed in a secondary process. ${ }^{5,6}$

The advance of X-ray scattering technique permits us to investigate the crystallization of polymer materials with increasing precision and time resolution. The established understanding concerning the relations among process parameters, mechanisms, and the resulting structure shall ultimately be exploited to tailor material properties by optimization of the industrial processes. Nevertheless, if isotropic materials are studied, then the information content of the scattering pattern is low, and simple models must be employed in the analysis. Studying the isothermal crystallization of PP from a quiescent, isotropic PP melt Albrecht and $\mathrm{Strobl}^{7}$ shows that the isotropic small-angle X-ray scattering (SAXS) can only be fitted by a "stack of crystalline lamellae" in the first few minutes after a shallow quench (i.e., high crystallization temperature). This scattering has also been observed by the group of Ryan ${ }^{8,9}$ but has been related to a spinodal decomposition of the melt because no discrete WAXS has been simultaneously observed. Other authors ${ }^{10,11}$ observe simultaneous growth of discrete WAXS and SAXS. They conclude that a nucleation-and-growth process is dominating crystallization. In this context, the question has been raised of whether the detection limit ${ }^{9,11,12}$ of common WAXS detectors was sufficient to capture the start of crystallite growth. In a very recent paper, ${ }^{13}$ the nucleation-and-growth mechanism

* Corresponding author. E-mail: norbert.stribeck@desy.de. Tel: +4940-42838-3615. Fax: +49-40-42838-6008.

University of Hamburg.

HASYLAB at DESY. has been affirmed by a crystallization study of isotropic PP in which the authors present data from a medium-deep quench to $145^{\circ} \mathrm{C}$. They report that other experimental conditions give essentially the same results.

Therefore, it appears to be suggestive to investigate crystallization at several crystallization temperatures, to use anisotropic samples (to avoid solid-angle smearing of discrete features), to record both SAXS and WAXS, and to elaborate a method that can be applied to anisotropic WAXS patterns and can return a well-founded measure of crystallinity. For this purpose, we choose materials that crystallize in the uniaxially oriented state. Oriented crystallization of polymers can be achieved by cautiously melting highly oriented materials with fiber symmetry to preserve the orientation memory ${ }^{14,15}$ of the polymer network or by shearing ${ }^{16-20}$ the melt. The indicated orientation-memory or self-nucleation effect has frequently been discussed, ${ }^{21-24}$ and the memory has been attributed to bundles of extended chain segments that are thought to be either thin rigid "shish" crystallites or noncrystalline chain bundles whose orientation persists on a time scale on which chain entanglements do not move. With increasing melt-annealing temperature and time, ${ }^{15}$ the oriented quiescent melt turns into an isotropic quiescent melt. We say that we have started from an oriented quiescent melt whenever oriented crystallites are emerging from a molten sample that does not show scattering. Oriented quiescent melts have been observed ${ }^{15}$ in PP between 170 (melt-annealing time $\left.t_{\mathrm{ma}} \geq 4 \mathrm{~min}\right)$ and $177^{\circ} \mathrm{C}\left(t_{\mathrm{ma}} \leq 4 \mathrm{~min}\right)$. These temperatures are far below the equilibrium melting temperature $\left(212{ }^{\circ} \mathrm{C}\right)$ of isotactic PP. ${ }^{25}$ Therefore, either to indicate remnant crystallites or to determine the onset of crystallization, quantitative parameters that are proportional to crystallinity or to crystallite size are determined and plotted as a function of the elapsed time.

Two years ago, we published a time-resolved SAXS study on the crystallization mechanisms in oriented isotactic PP. ${ }^{15} \mathrm{We}$ reported that after shallow quench $\left(155\right.$ and $\left.150{ }^{\circ} \mathrm{C}\right)$, the first discrete SAXS develops into meridional streaks. After medium and deep quench, the first discrete SAXS patterns conform to both the model of a cross-hatched ${ }^{4,5,7,26}$ lamellar structure and Strobl's block structure. ${ }^{27,28}$ The ambiguity results from Babinet's theorem. Nevertheless, from the continued evolution of the scattering patterns into a pattern of oriented lamellae, we 


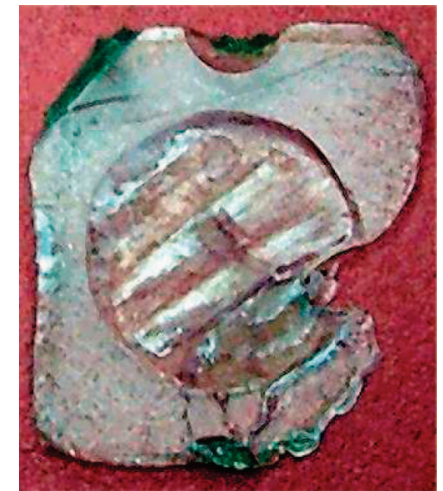

Figure 1. HEPP film after a melting and recrystallization experiment. Aluminum foils are removed.

have concluded that a merging of the blocks into lamellae is more probable than a disruption of cross-lamellae in a quiescent process.

Following the suggestions of the referees of our SAXS study, ${ }^{15}$ we now have also monitored the isothermal crystallization of the same commercial material by WAXS. Here we present the results and discuss them in connection with the results of the SAXS study. In the course of the WAXS experiments, several thousand 2D WAXS patterns have been recorded. Data evaluation of such an amount of data can be accomplished by either a simple manual analysis method or automatic processing of the diffraction patterns. We have tested both possibilities. After one of us had deduced an exact equation for the determination of the fiber tilt angle, ${ }^{29}$ an automatic quantitative evaluation procedure for fiber patterns ${ }^{30}$ became possible. By means of this procedure, one can easily track and compensate the tumbling of the fiber axis during the experiment. Moreover, materials parameters that are well-founded in scattering theory can be computed and tracked. Ultimately, we track the weight crystallinity and the extension of the crystallites for the two differently oriented sets of crystallites.

\section{Experimental Section}

2.1. Material. Commercial hard-elastic ${ }^{31,32}$ polypropylene (HEPP) film (CelGard-PP, lot no. 884, as extruded by Hoechst-Celanese) of $25 \mu \mathrm{m}$ thickness is studied. The films are produced ${ }^{33}$ from the PP grade Hercules Profax 6301 with a melt index of $15.0\left(230^{\circ} \mathrm{C}\right)$ ASTM-D-1238. The weight-average molecular mass of the grade is $\bar{M}_{\mathrm{w}}=128000 \mathrm{~g} / \mathrm{mol}$. The polydispersity of the material is $\bar{M}_{\mathrm{w}} /$ $\bar{M}_{\mathrm{n}}=4$. Sixteen sheets are stacked under consideration of their high uniaxial orientation, covered by aluminum foil $(20 \mu \mathrm{m}$ thickness), and fixed in a frame sample holder.

2.2. Wide-Angle $X$-ray Scattering Measurements. WAXS is performed in the synchrotron beamline A2 at HASYLAB, Hamburg, Germany. The wavelength of the X-ray beam is $0.15 \mathrm{~nm}$, and the sample-detector distance is $79 \mathrm{~mm}$. Scattering patterns are collected by a 2D position-sensitive marccd 165 detector (mar research, Norderstedt, Germany) in binned $1024 \times 1024$ pixel mode (158.3 $\mu \mathrm{m}$ quadratic pixel size). During the experiments, series of scattering patterns are recorded. The cycle time is either $30 \mathrm{~s}(20$ $\mathrm{s}$ exposure) or $8 \mathrm{~s}$ (4 $\mathrm{s}$ exposure).

2.3. Temperature Programs. The frames, each containing a stack of foils, are mounted in a furnace equipped with two heating cartridges and air cooling that is provided by HASYLAB. Hardware and temperature programs are the same as those in a previous SAXS study..$^{15}$ The samples are rapidly heated to $150^{\circ} \mathrm{C}$. Then, the heating rate is lowered to $2{ }^{\circ} \mathrm{C} / \mathrm{min}$ to approach a melt-annealing temperature of $173{ }^{\circ} \mathrm{C}$ cautiously. The melt-annealing temperature is kept for $4 \mathrm{~min}$. After this time, the sample is cooled to the crystallization temperature $\left(150,145\right.$, or $\left.140{ }^{\circ} \mathrm{C}\right)$ within $1 \mathrm{~min}$. The temperature is measured in the sample holder. Therefore, the sample temperature may be lower than the measured temperature. Figure 1 shows a sample after one of the experiments. The samples are warping during the experiments.

\section{Data Analysis}

All scattering patterns show the $\alpha_{2}$-modification of PP. ${ }^{34}$ The WAXS data are analyzed in two different ways, which are sketched in Figure 2. Figure 2a demonstrates a simple 1D analysis that makes only rudimentary use of scattering theory. Figure $2 b$ sketches a 3D analysis method. Here the intensity of the fiber pattern (Figure 2a) is mapped into reciprocal space before peaks are isolated and analyzed by integration in reciprocal space. The change of color in the pseudocolor images from Figure $2 \mathrm{a}$ to Figure $2 \mathrm{~b}$ indicates the intensity correction by consideration of the fiber mapping, ${ }^{29,30}$ the absorption, and the polarization factor. In principle, only the $3 \mathrm{D}$ evaluation is exact. Nevertheless, Figure $2 \mathrm{~b}$ reveals a general problem of fiber diffraction. A blind zone extends across the meridian ( $s_{3}$ axis). It is a function of the fiber tilt angle, $\beta$.

3.1. One-Dimensional Wide-Angle $X$-ray Scattering Trend Analysis. The 1D analysis ignores an essential part of the scattering theory to provide a trend analysis of the crystal structure evolution. As indicated in Figure 2a, two scattering curves (sections) are cut from the raw diffraction patterns. The first cut runs along the equator passing the three strongest reflection peaks of PP. It serves the analysis of the $c$-oriented crystallites. The second cut runs through the maximum of the almost-meridional 110 reflection for an analysis of the crystallites whose $a^{*}$ axes are in the fiber direction. As the orientation of the fiber axis with respect to the X-ray beam tumbles during the experiment, the reflection maximum moves on a circle. ${ }^{29,35}$ It can even vanish at the meridian.

Without correction of the intensity, the cuts are rescaled (e.g., $s_{12}=(2 / \lambda) \sin \left(0.5 \arctan \left(p_{12} / R\right)\right.$, where $R$ is the sample-detector distance) from the detector coordinate system, $p$, to units of the scattering vector, $s$, in the direction of the cut. Therefore, for the equatorial and quasi-meridional directions, curves $I_{\mathrm{e}}\left(s_{12}\right)$ and $I_{\mathrm{m}}\left(s_{*}\right)$ are obtained, respectively. Here $s=|s|=(2 / \lambda) \sin \theta$ is the modulus of the scattering vector, which is a function of the scattering angle, $2 \theta$, and the X-ray wavelength, $\lambda$. Let the Cartesian coordinates of the scattering vector be $s=\left(s_{1}, s_{2}, s_{3}\right)$. Then, the transverse (i.e., equatorial) component of the scattering vector is defined as $s_{12}=\left(s_{1}^{2}+s_{2}^{2}\right)^{1 / 2}$. The direction $s_{*}$ of the quasi-meridional component depends on the studied material. In each cut, the 110 peak is separated from its background, resulting in the peak curves $I_{(110)}\left(s_{12}\right)$ and $I_{(110)}^{*}(s *)$, respectively. From the peak curves, $1 \mathrm{D}$ reflection strengths

$$
S_{1}=\int I_{(110)}\left(s_{12}\right) \mathrm{d} s_{12}
$$

and

$$
S_{1}^{*}=\int I_{(110)}^{*}\left(s_{*}\right) \mathrm{d} s_{*}
$$

are integrated, which describe the trend of crystallinity evolution during the experiment. Additionally, 1D integral breadths

$$
B_{1}=\frac{S_{1}}{\max \left(I_{(110)}\left(s_{12}\right)\right)}
$$

and

$$
B_{1}^{*}=\frac{S_{1}^{*}}{\max \left(I_{(110)}^{*}\left(s_{*}\right)\right)}
$$

are computed, from which quantities $b_{1}=1 / B_{1}$ and $b_{1}^{*}=1 / B_{1}^{*}$ are obtained, which are used as measures for the lateral extension of the $c$ - and $a^{*}$-oriented crystallites. ${ }^{36}$ The required manual 

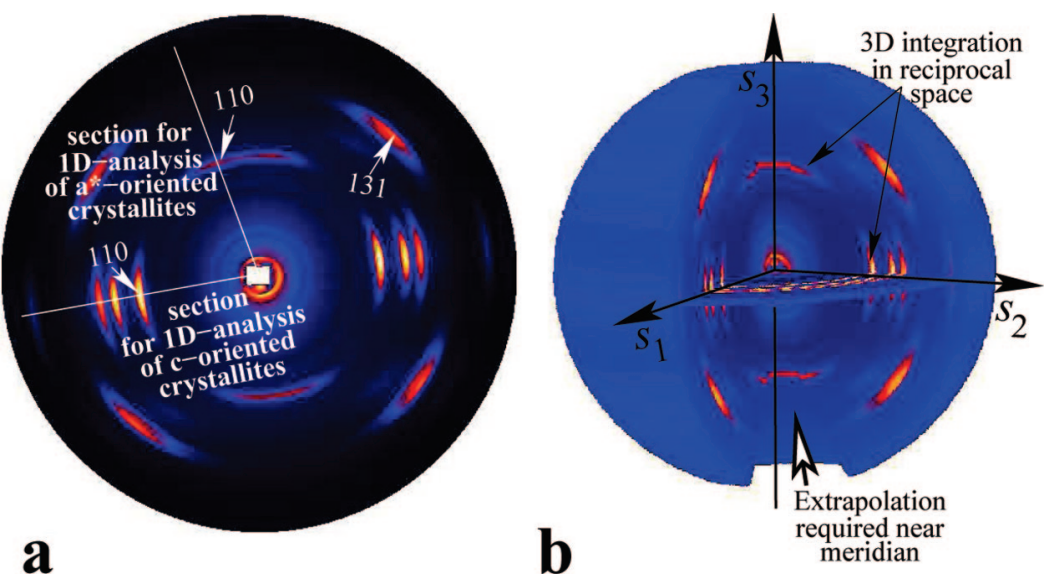

Figure 2. Variants of fiber diffraction analysis for hard-elastic polypropylene. (a) 1D analysis in the raw measured pattern by excision of curves and integration yielding integral and breadth of the 110 reflections. (b) $3 \mathrm{D}$ analysis by mapping the complete pattern into reciprocal space and $3 \mathrm{D}$ integration. $s_{1}, s_{2}$, and $s_{3}$ are the components of the scattering vector, $s$.

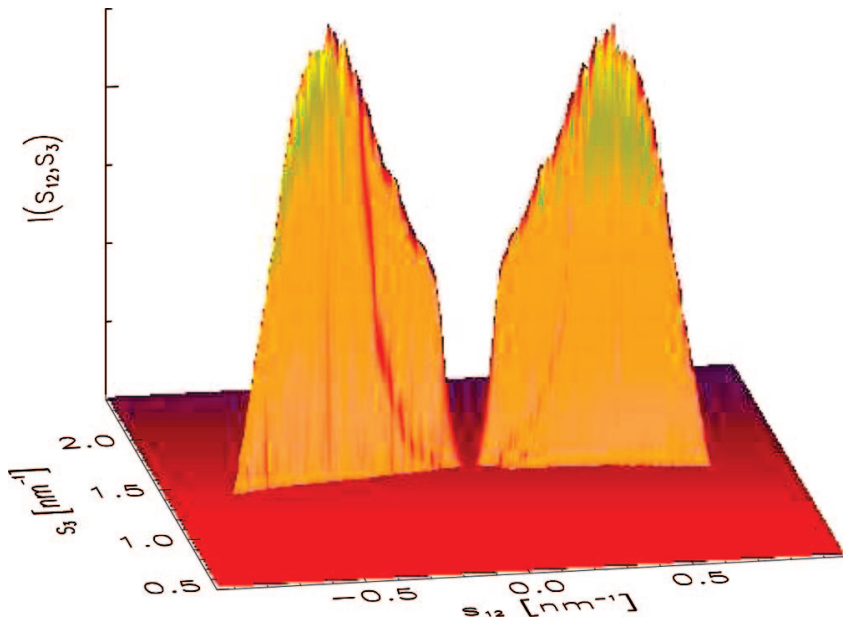

Figure 3. Typical quasi-meridional 110 peaks of HEPP as extracted (during recrystallization $24 \mathrm{~min}$ after quenching to $150^{\circ} \mathrm{C}$ ). Displayed region: $-1 \mathrm{~nm}^{-1}<s_{12}<1 \mathrm{~nm}^{-1} ; 0.5 \mathrm{~nm}^{-1}<s_{3}<2.5 \mathrm{~nm}^{-1}$.

operations comprise proper rotation and background subtraction. They have to be carried out twice for each diffraction pattern. The processing of several thousand patterns collected in 1 day has been carried out in 3 months by 1 person.

3.2. Three-Dimensional Wide-Angle X-ray Scattering Data Analysis. 3D WAXS data analysis first requires us to map $^{30,35,37,38}$ the recorded pattern from the detector plane into reciprocal space. During mapping, the fiber rotation angle and the fiber tilt angle are automatically determined. ${ }^{29}$ The thermal expansion of crystallites is not considered. Therefore, the distance of the tumbling sample from the detector is individually computed for each diffraction pattern from the tabulated ${ }^{34}$ positions of the reflections of the $\alpha_{2}$ modification of PP. After the mapping, the recorded diffraction data fill a nearly spherical volume (Figure 2b) in reciprocal space, except for a blind volume that extends along the meridian. Mapping of one pattern takes $2 \mathrm{~s}$. The extraction and analysis of two 110 peaks require $20 \mathrm{~s}$ for each pattern. Most of the machine time is spent in a $2 \mathrm{D}$ background extrapolation routine. The source code of the computer programs is freely available. ${ }^{39}$ The core procedures are wf_spotana.pro and wf_merfit.pro.

After the fiber mapping, even the quasi-meridional 110 reflection is always found at the same place. Therefore, the manual effort is limited to the definition of two regions of interest (ROI) that are valid for all patterns in all experiments. In each of these regions, a single peak is found. Before the analysis of the quasi-meridional peak, the blind gap must be filled by extrapolated data (wf_merfit.pro). An algorithm based on radial basis functions ${ }^{40}$ is applied, which returns a smooth background. Nevertheless, it is not capable of reconstructing the central reflection intensity (cf. Figure 3 ). The error introduced by this shortcoming is discussed in the following section. Finally, each reflection spot is analyzed by the procedure wf_spotana.pro. The analysis employs standard methods ${ }^{41,42}$ of digital image processing. The application of the Sobel operator localizes the reflection inside its ROI. The opening operator makes sure that its center and the outer falloff region are accounted for. The remnant pixels of the ROI are background and are used for background extrapolation. The background is subtracted, and separated peaks $I_{(110)}(s)$ and $I_{(110)}^{*}(s)$ are obtained. For the computation of the total reflection intensities

$$
S=2 \pi \iint s_{12} I_{(110)}\left(s_{12}, s_{3}\right) \mathrm{d} s_{12} \mathrm{~d} s_{3}
$$

and

$$
S^{*}=2 \pi \iint s_{12} I_{(110)}^{*}\left(s_{12}, s_{3}\right) \mathrm{d} s_{12} \mathrm{~d} s_{3}
$$

fiber symmetry is exploited. According to Ruland, ${ }^{43,44}$ these integrals are proportional to the weight crystallinity of the perfect crystals in the irradiated volume. Nevertheless, because a small fraction of the meridional intensity is invisible and not reconstructed, the determined value of $S^{*}$ is somewhat too small.

For the determination of the radial integral breadth, $B$, first, radial scattering curves of the extracted peak

$$
I_{(110)}(s)=2 \pi \int s_{12} I_{(110)}(s, \phi) \mathrm{d} \phi
$$

are computed by azimuthal integration $\left(\cos \phi=s_{12} / s\right)$. From $I_{(110)}(s)$

$$
B=\int I_{(110)}(s) \mathrm{d} s / \max \left(I_{(110)}(s)\right)
$$

is obtained. Analogously, the breadth $B^{*}$ of the $a^{*}$-oriented crystallites is computed. From the Fourier transform breadth theorem, ${ }^{36}$ it follows that $b=1 / B$ and $b^{*}=1 / B^{*}$ are the integral breadths of the lateral extensions for the effective shape function of the crystallites with $c$-axis orientation and $a^{*}$-axis orientation, respectively. After crystallization has started, peaks emerge out of the noisy background. The procedure can separate narrow peaks (e.g., $a^{*}$-set reflections) from the noise at already lower peak integrals than broader ones.

Although the shape function ${ }^{36}$ of the crystallites comprises several factors, we are not trying to separate these factors but instead call the inverse integral breadths "minimum crystallite 


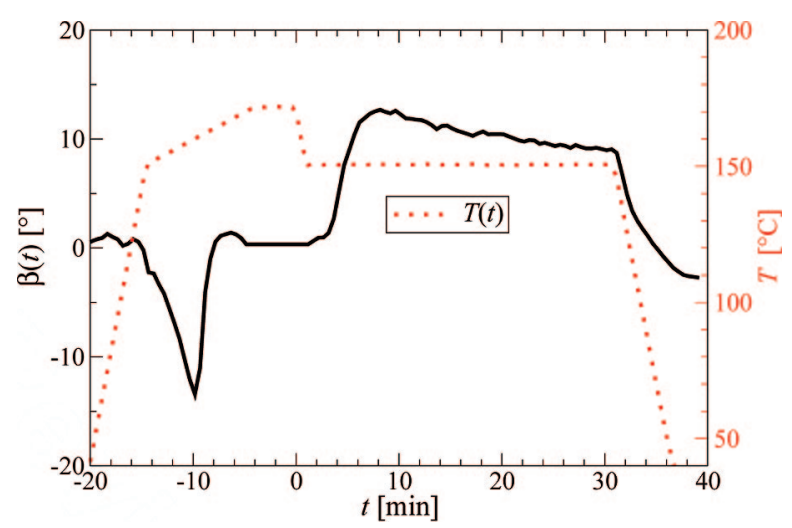

Figure 4. Tilt-angle tracking curve, $\beta(t, T)$, from the automatic fiber mapping procedure in an experiment with long exposure of the patterns $(20 \mathrm{~s})$ in which $\beta$ changes considerably (HEPP; melt annealing at 171 ${ }^{\circ} \mathrm{C}$ and recrystallization at $150{ }^{\circ} \mathrm{C}$ ).

extension". We consider this to be permitted in this study because here the shape of the corresponding curves is used, first, to compare results of different data extraction methods and, second, to detect remnant crystals in the early stage of crystallization after quenching from the rather cool melt. Therefore, we do not discuss effects ${ }^{36,45,46}$ that additionally broaden the reflections such as the natural line width, strain broadening, and crystallite imperfections.

3.3. Error upon Extraction of the Meridional Peak. A typical extracted meridional peak, $I_{(110)}^{*}\left(s_{12}, s_{3}\right)$, is shown in Figure 3 . The observable ridge on the reflection is an artifact that is caused by inaccuracy of the automatic fiber mapping concerning the center and rotation angle of the raw diffraction pattern. The central break in is caused by improper extrapolation into the blind gap at the meridian. Although the computed total reflection intensity, $S^{*}$, is somewhat too small, the error is moderated by the fact that the missing intensity is close to the meridian; that is, it is distributed on circles with small radii (cf. Figure $2 \mathrm{~b}$ ). Therefore, the intensity from the underestimated region contributes with low weight only. In our experiments, it appears to be more grave that the underestimation is a function of the varying tilt angle, $\beta$. Underestimation becomes negligible whenever $\beta=\theta_{110}$ passes half of the scattering angle of the probed meridional reflection because in this moment, the symmetrical reflection geometry ${ }^{36}$ is realized.

\section{Results and Discussion}

4.1. Tracking of the Fiber Tilt Angle under Thermal Load. The studied uniaxially oriented film samples soften and warp upon heating (cf. Figure 1). Therefore, considerable tumbling of the fiber axis in the small volume irradiated by the $\mathrm{X}$-ray beam is expected. The fiber mapping procedure ${ }^{29,30}$ automatically tracks both the fiber rotation and the tilt angle, $\beta$. On the basis of these parameters, the data are corrected. The statistical error of the tilt-angle determination can be estimated from the statistical noise on the tilt-angle curve, $\beta(t)$, of the experiments.

In two of the eight experiments carried out, $\beta(t)$ stays close to $0^{\circ}$. In two other experiments, extreme changes are observed. The corresponding curves are documented in Figures 4 and 5. Figure 4 shows $\beta(t, T)$ of an experiment in which very good signal-to-noise ratio in the patterns has been achieved by exposing for $20 \mathrm{~s}$ (cycle time: $30 \mathrm{~s}$ ). The tracking curve appears to be rather smooth, demonstrating the reliability of the tiltangle determination. Figure 5 documents the most extreme tiltangle variation from the experiments with $4 \mathrm{~s}$ exposure $(8 \mathrm{~s}$ cycle time). The increased noise on the tracking curve is a result of the inferior signal-to-noise ratio in the diffraction patterns.

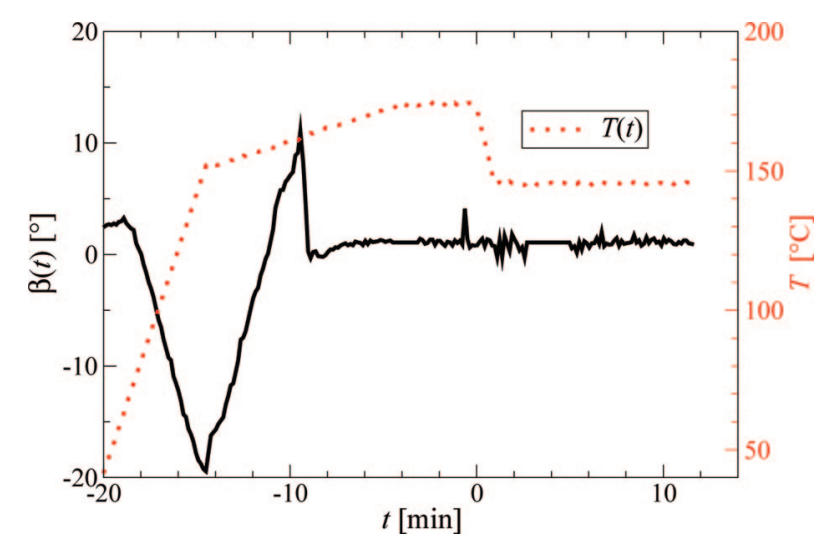

Figure 5. Tilt-angle tracking curve, $\beta(t, T)$, from the automatic fiber mapping procedure in an experiment with short exposure of the patterns $(4 \mathrm{~s})$ in which $\beta$ changes considerably (HEPP; melt annealing at 173 ${ }^{\circ} \mathrm{C}$ and recrystallization at $145^{\circ} \mathrm{C}$ ).

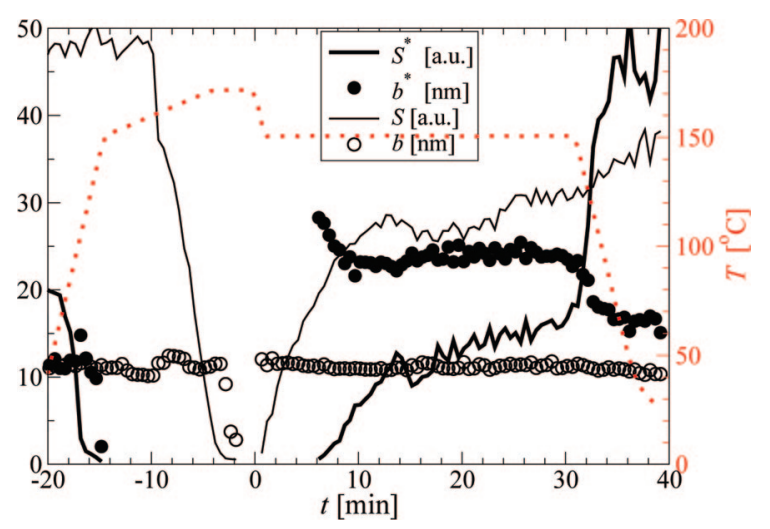

Figure 6. Three-dimensional analysis of the 110 reflections of HEPP under thermal load. Melting at $171{ }^{\circ} \mathrm{C}$ and isothermal crystallization at $150{ }^{\circ} \mathrm{C}$. Dotted curve: temperature, $T(t)$. Solid lines: peak integrals in reciprocal space, $S$ and $S^{*}$, of $c$ - and $a^{*}$-oriented crystals, respectively. Symbols: minimum lateral crystallite sizes (integral breadths), $b$ and $b^{*}$.

4.2. Isothermal Crystallization at $150{ }^{\circ} \mathrm{C}$. Exactly as in a previous SAXS study, ${ }^{15}$ the HEPP sample has been melt annealed at a furnace temperature of $171^{\circ} \mathrm{C}$ for $4 \mathrm{~min}$ and quenched to $150{ }^{\circ} \mathrm{C}$ for isothermal crystallization. Figure 6 exhibits the results of the $3 \mathrm{D}$ reflection analysis in reciprocal space. Figure 7 shows the respective curves from the 1D WAXS trend analysis. The comparison of the two Figures demonstrates that the essential features are recognized in both kinds of evaluation: early melting and late crystallization of the $a^{*}$ oriented set become apparent in both the 1D integrated peak intensity, $S_{1}^{*}(t)$, and the $3 \mathrm{D}$ integral in reciprocal space, $S^{*}(t)$. Also, the doubling of the size $b^{*}$ of the $a^{*}$-set crystallites during crystallization from the quiescent melt is demonstrated in both analyses. Similarly, both evaluations show that the amount of $a^{*}$ crystallites considerably increases during the quench after the isothermal phase (for $t>30 \mathrm{~min}$ ) but that the new crystallites do not grow to be big.

On the contrary, the evaluation of sections $S_{1}(t)$ pretends that for $t>30 \mathrm{~min}$ there is a steep increase in the $c$ crystallites, whereas the weight crystallinity $S(t)$ increases only gradually. The interpretation of the integral crystallite sizes, $b^{*}$, suggests that the first $a^{*}$ crystallites appear to be big. On the contrary, the simple $1 \mathrm{D}$ analysis $\left(b_{1}^{*}\right)$ suggests that the first $a^{*}$-oriented crystallites were small. For the other studied experiments, both the general agreement and the individual discrepancies of the $1 \mathrm{D}$ and the 3D method are similar. 


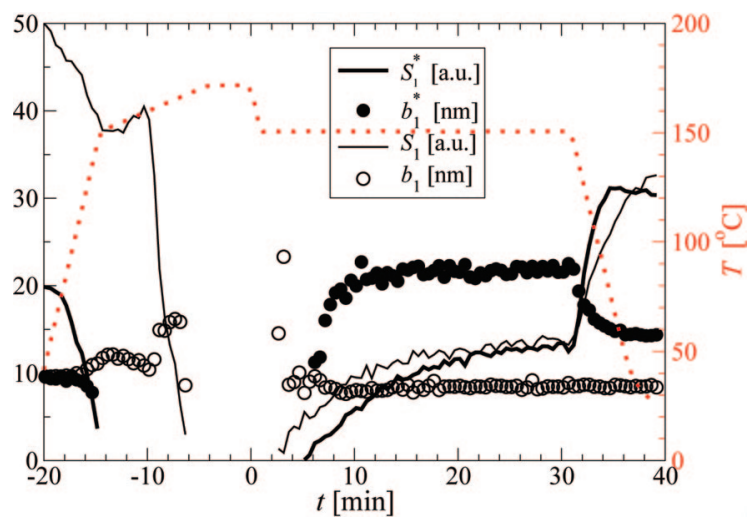

Figure 7. One-dimensional analysis of sections cut through the 110 reflections of HEPP under thermal load. Melting at $171{ }^{\circ} \mathrm{C}$ and isothermal crystallization at $150{ }^{\circ} \mathrm{C}$. Dotted curve: temperature, $T(t)$. Solid lines: peak strengths from the sections, $S_{1}$ and $S_{1}^{*}$, of $c$ - and $a^{*-}$ oriented crystals, respectively. Symbols: lateral crystallite sizes, $b_{1}$ and $b_{1}^{*}$.

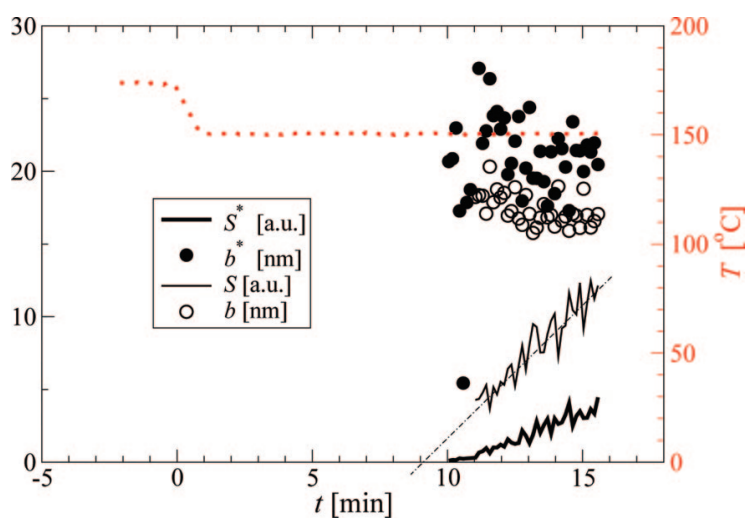

Figure 8. Three-dimensional analysis of the 110 reflections of HEPP under thermal load. Melting at $173{ }^{\circ} \mathrm{C}$ and isothermal crystallization at $150{ }^{\circ} \mathrm{C}$. Dotted curve: temperature, $T(t)$. Solid lines: peak integrals in reciprocal space, $S$ and $S^{*}$, of $c$ - and $a^{*}$-oriented crystals, respectively. Symbols: lateral crystallite sizes (integral breadths), $b$ and $b^{*}$.

4.3. Remanent Crystal Nuclei. In Figure 6, the trend of the strength $S(t, T)$ close to $t=0$ is indicative of remanent nuclei of the $c$ set. For other (not presented) experiments with a meltannealing temperature of $171{ }^{\circ} \mathrm{C}$, remanent crystal reflections have even been observed. Therefore, in a second series of experiments, the melt-annealing temperature is increased to 173 ${ }^{\circ} \mathrm{C}$. The time-resolution is enhanced ( $8 \mathrm{~s}$ cycle time) at the expense of the background noise. The amount of collected data is kept low by lowering the interval in which WAXS monitoring is turned on. Figure 8 shows the result for the crystallization at $150{ }^{\circ} \mathrm{C}$. A latency period of about $10 \mathrm{~min}$ that can now be read from the peak-integral curves confirms the suspicion. Nuclei must have been present after quenching from the cooler melt, although in the earlier SAXS study, ${ }^{15}$ a measured furnace temperature of $171{ }^{\circ} \mathrm{C}$ had proven to be sufficient.

In many studies, WAXS patterns are not analyzed by peak integration but by visual inspection. Figure 9 shows mapped diffraction patterns in a pseudocolor intensity scale that brings out even weak reflections in print. Only an isotropic amorphous halo is observed $3.2 \mathrm{~min}$ after the quench (Figure 9a). Therefore, visual inspection and peak integration are still in agreement. After $3.5 \mathrm{~min}$ (Figure 9b), the amorphous halo has already somewhat retracted from the equator, where a weak 110 reflection is now perceptible. The first indication of $a^{*}$-oriented crystallites is found after 6.5 min (Figure 9c) and is clearly visible after $7 \mathrm{~min}$. Throughout the interval covered in Figure

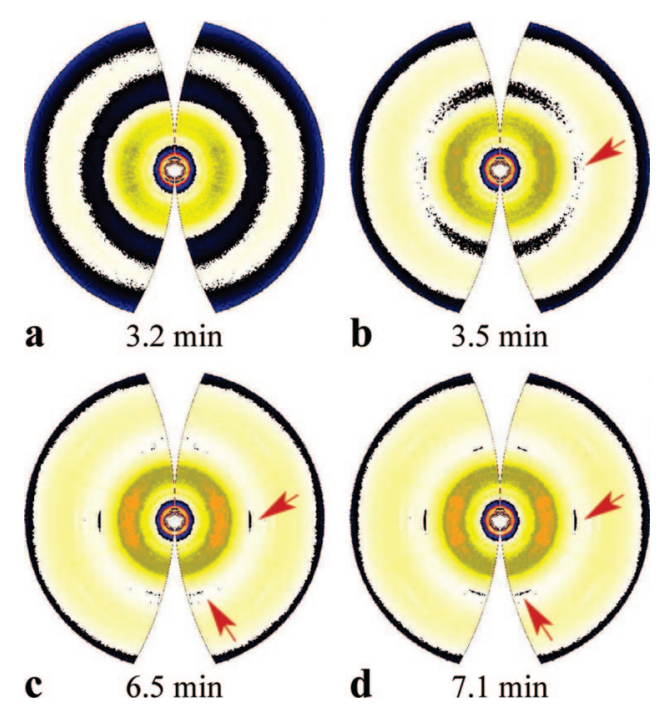

Figure 9. Visual inspection of the crystalline reflections of HEPP under thermal load. Isothermal crystallization after melting at $173{ }^{\circ} \mathrm{C}$. Indicated is the time after quenching to $150{ }^{\circ} \mathrm{C}$.

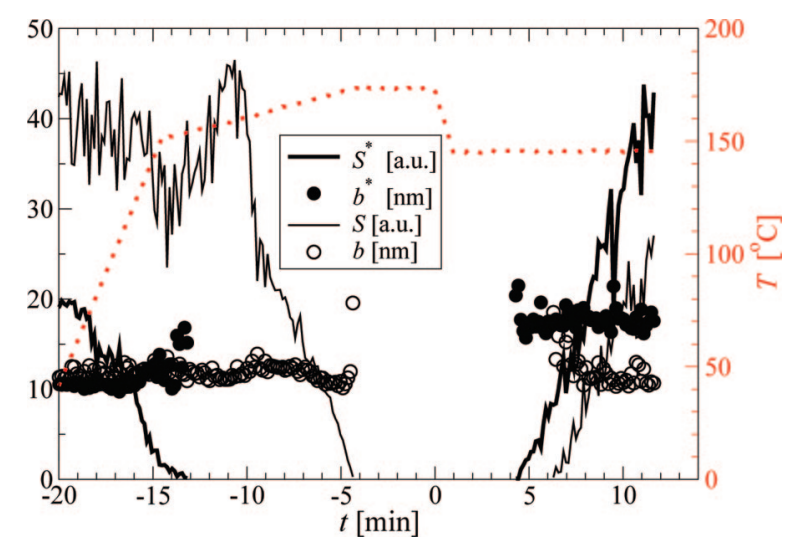

Figure 10. Three-dimensional analysis of the 110 reflections of HEPP under thermal load. Melting at $173^{\circ} \mathrm{C}$ and isothermal crystallization at $145^{\circ} \mathrm{C}$. Dotted curve: temperature, $T(t)$. Solid lines: peak integrals in reciprocal space, $S$ and $S^{*}$, of $c$ - and $a^{*}$-oriented crystals, respectively. Symbols: lateral crystallite sizes (integral breadths), $b$ and $b^{*}$.

9, the 3D-peak analysis does not return a significant integral. The corresponding weight crystallinity is still very small.

Assuming that the weight crystallinity of the completely crystallized material is $50 \pm 20 \%$, the comparison of the low and the high end of the peak-strength curves shows that the automatic peak analysis logs in at weight crystallinities that are close to $1 \%$. The fact that reflections are visually conceivable long before that threshold is surpassed indicates that relevant weight crystallinities (in the sense of materials science) are generated much later than visual inspection presumes. The evolution of crystallinity is discussed in Section 4.6.

4.4. Isothermal Crystallization at $145{ }^{\circ} \mathrm{C}$. Figure 10 shows the evolution of crystallites during melting and crystallization. The automatic peak analysis catches the strength, $S^{*}(t)$, of the $a^{*}$ set 4 min after the quench. $S(t)$ starts 6 min after quenching to $145^{\circ} \mathrm{C}$.

Again, the peaks are detected earlier by visual inspection. The material is still completely amorphous $3.1 \mathrm{~min}$ after quenching (Figure 11a). Both the quasi-meridional 110 reflection of the $a^{*}$ set and the 131 reflection are visible $8 \mathrm{~s}$ after that (Figure 11b). At $t=3.5 \mathrm{~min}$ (Figure 11d), even the equatorial 110 peaks are observable. In HEPP crystallized isothermally at $145{ }^{\circ} \mathrm{C}$ from the quiescent melt, the $a^{*}$-oriented crystallites appear earlier than the $c$ crystallites. 

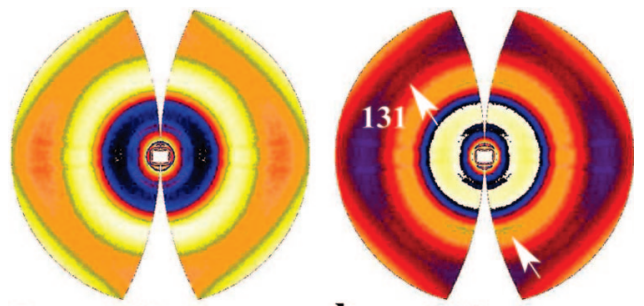

a

$3.10 \mathrm{~min}$

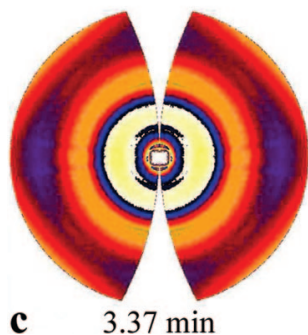

b

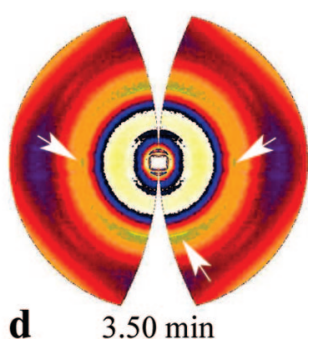

Figure 11. Visual inspection of the crystalline reflections of HEPP under thermal load. Isothermal crystallization after melting at $173{ }^{\circ} \mathrm{C}$. Indicated is the time after quenching to $145^{\circ} \mathrm{C}$. The horizontal distortion in the center results from adhesive tape holding the beam stop.

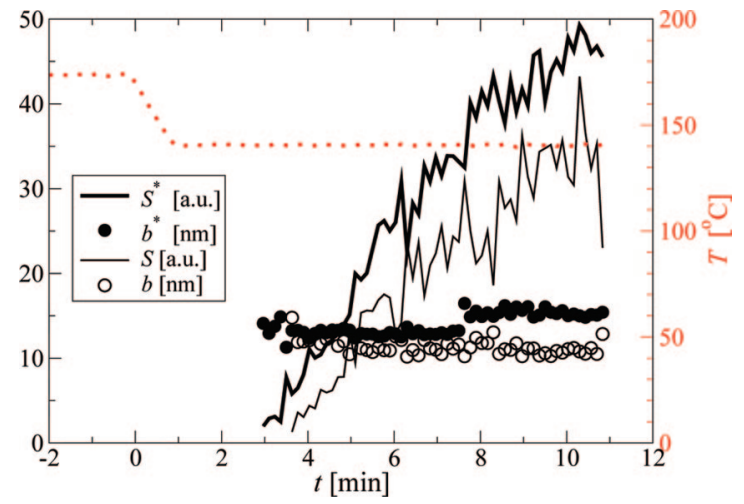

Figure 12. Three-dimensional analysis of the 110 reflections of HEPP under thermal load. Melting at $173^{\circ} \mathrm{C}$ and isothermal crystallization at $140{ }^{\circ} \mathrm{C}$. Dotted curve: temperature, $T(t)$. Solid lines: peak integrals in reciprocal space, $S$ and $S^{*}$, of $c$ - and $a^{*}$-oriented crystals, respectively. Symbols: lateral crystallite sizes (integral breadths), $b$ and $b^{*}$.

4.5. Isothermal Crystallization at $140{ }^{\circ} \mathrm{C}$. Figure 12 shows the evolution after the quench from 173 to $140^{\circ} \mathrm{C}$. The process is faster than at $145^{\circ} \mathrm{C}$. Now the quasi-meridional peaks of the $a^{*}$ set become separable after $3 \mathrm{~min}$. The equatorial $c$-set reflection is first captured after $3.5 \mathrm{~min}$. The minimum extensions, $b$ and $b^{*}$, of both kinds of crystallites are almost identical in the beginning. $b^{*}$ increases from 12 to $15 \mathrm{~nm} 7.5 \mathrm{~min}$ after the quench. Again, the eye detects the crystalline reflections earlier. Figure 13a still shows an amorphous melt after $1.5 \mathrm{~min}$. Both the reflection on the first layer line (arrow labeled "131") and the quasi-meridional $a^{*}$-related reflection become visible $16 \mathrm{~s}$ later (Figure 13b). It takes another $40 \mathrm{~s}$ before the $c$-set crystallites are distinguished (Figure 13d). Here all three strong equatorial reflections of PP even show up simultaneously.

4.6. Evolution of Weight Crystallinities. Figure 14 shows the evolution of the weight crystallinities of the two sets of crystallites at the three different crystallization temperatures. In this plot, all 3D peak-integral curves are normalized in the same way. Therefore, they can be compared relative to each other. As shown by Ruland, ${ }^{43}$ such reflection integrals that are complete in reciprocal space are proportional to the weight crystallinity of the perfect crystallites that cause the reflections.

4.7. Latency Periods. In Figure 14, visually determined latency periods between the beginning of the quench and the

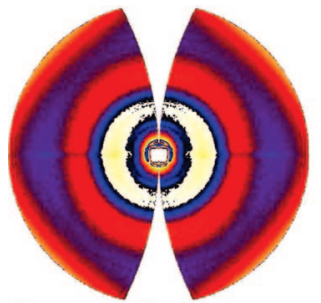

a

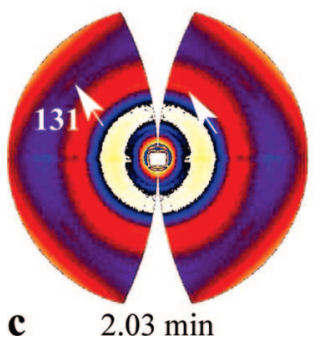

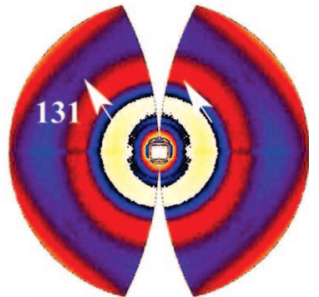

b $\quad 1.76 \mathrm{~min}$

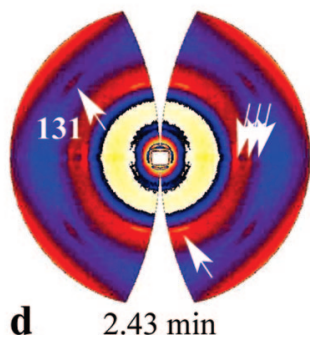

Figure 13. Visual inspection of the crystalline reflections of HEPP under thermal load. Isothermal crystallization after melting at $173{ }^{\circ} \mathrm{C}$. Indicated is the time after quenching to $140^{\circ} \mathrm{C}$. The horizontal distortion in the center results from adhesive tape holding the beam stop.

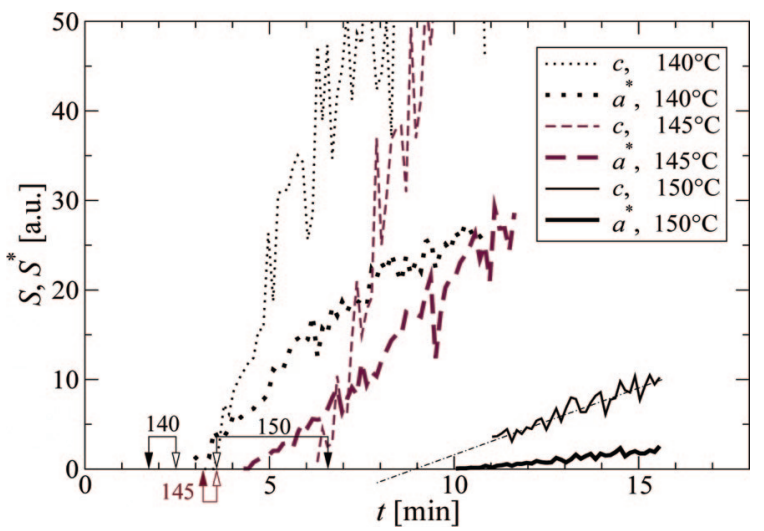

Figure 14. Evolution of relative weight crystallinities of the $a^{*}$-oriented crystallites (bold lines) and of the $c$-oriented crystallites (thin lines) during isothermal oriented crystallization of HEPP from a quiescent melt as a function of crystallization temperature. Double arrows indicate the first sighting of the $a^{*}$ set (full arrowhead) and the $c$ set (open arrowhead), respectively.

first observation of 110 reflections are indicated by double-head arrows. Full arrowheads mark $t_{v 0}^{*}$, that is, the first sighting of the quasi-meridional reflection of the $a^{*}$-oriented crystallite set. The open arrowhead indicates $t_{v 0}$, that is, the first observation of the equatorial reflection.

Latency periods $t_{0}^{*}$ and $t_{0}$ determined from the weightcrystallinity curves are defined by the conditions $S^{*}\left(t_{0}^{*}\right)=0$ and $S\left(t_{0}\right)=0$, respectively. At the crystallization temperature, $T_{\mathrm{c}}=$ $150{ }^{\circ} \mathrm{C}$, the value $t_{0}=9 \mathrm{~min}$ must be extrapolated (dasheddotted line) because the separation algorithm captures the peak rather late. Both kinds of latency periods increase in similar manner as a function of $T_{\mathrm{c}}$. At low $T_{\mathrm{c}}\left(140{ }^{\circ} \mathrm{C}\right)$, the latency periods of the $a^{*}$ set are shorter than that of the $c$ set. With increasing $T_{\mathrm{c}}$, both $t_{v 0}^{*}$ and $t_{0}^{*}$ increase more rapidly than $t_{v 0}$ and $t_{0}$. Finally, at $T_{\mathrm{c}}=150{ }^{\circ} \mathrm{C}$, the latency of the $c$ set becomes shorter than that of the $a^{*}$ set.

The Figure shows that $t_{v 0}<t_{0}$ and $t_{v 0}^{*}<t_{0}^{*}$ are always valid. The difference is considerable. It is significant because the peakintegration algorithm logs in at very low weight crystallinities for most of the peaks, and no steepening of the curves is observed close to the end of the latency periods. (The opposite is observed during second melting; cf. Figure 16 and the corresponding discussion.) 

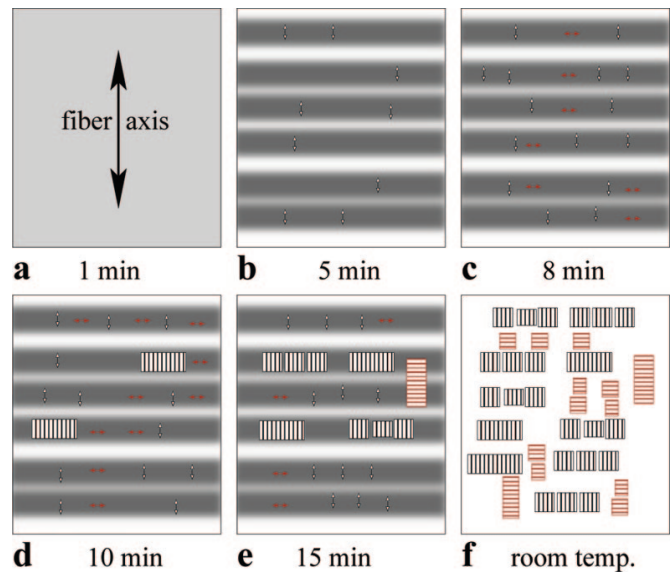

Figure 15. HEPP crystallizing from a quiescent, oriented melt at 150 ${ }^{\circ} \mathrm{C}$. (a) homogeneous melt, (b) decomposed melt with $c$-set nuclei (blocks), (c) melt with $c$-set and $a^{*}$-set nuclei, (d) primary $c$-set lamella, (e) $c$-set and primary $a^{*}$-set lamellae, and (f) cross-hatched structure.

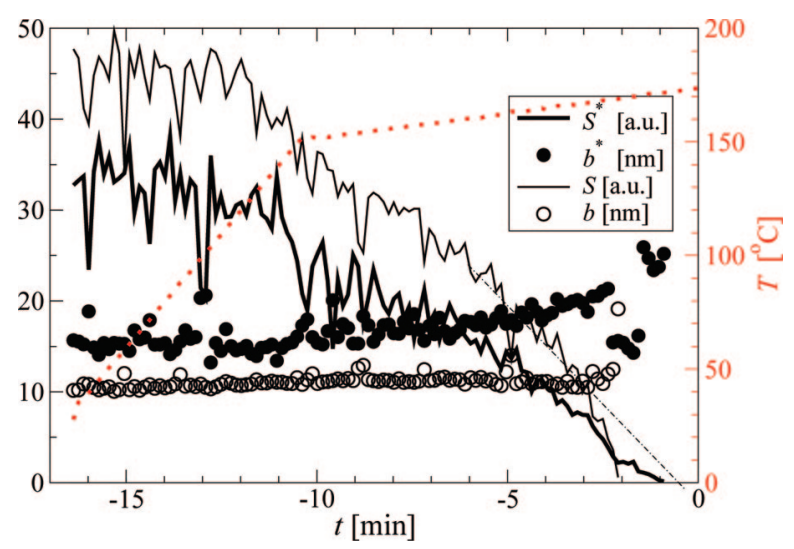

Figure 16. Three-dimensioanl analysis of the 110 reflections of HEPP under thermal load. Second melting after an isothermal crystallization experiment. Dotted curve: temperature, $T(t)$. Solid lines: peak integrals in reciprocal space, $S$ and $S^{*}$, of $c$ - and $a^{*}$-oriented crystals, respectively. Symbols: lateral crystallite sizes (integral breadths), $b$ and $b^{*}$.

Because in the intervals $\left[t_{v 0}, t_{0}\right]$ and $\left[t_{v 0}^{*}, t_{0}^{*}\right]$ the weight crystallinity increases much more slowly than thereafter, we propose that here a crystallization mechanism of its own is active, which is dominated by equilibrium between wax and wane of crystallites. This means that we are, in fact, observing nuclei. If a critical density of nuclei would induce the growth of stable crystals, then the average distance between the nuclei would become the average distance between Strobl's blocks. ${ }^{27,28}$

4.8. Crystallization Velocities. The slopes of the curves from Figure 14 are proportional to the crystallization velocities and can be compared with respect to each other. The graphs exhibit the well-known fact that all velocities slow down as the undercooling decreases. Moreover, the initial velocity of the $a^{*}$-set crystallites is less than half of the velocity of the $c$-set crystallites.

4.9. Combining WAXS and SAXS Results. In the following discussion, we assume that the sample temperature curves in the WAXS experiments are sufficiently close to those in the SAXS experiments. In the isotropic and shear-induced studies on PP crystallization cited in the introduction, similar latency periods and general features are reported as a function of crystallization temperature. Nevertheless, only combined SAXS - WAXS experiments with 2D detectors and high time resolution will help to remove the uncertainty.
4.9.1. Crystallization at $150^{\circ} \mathrm{C}$. In the SAXS study, ${ }^{15}$ (Figure 5) strong meridional scattering has been detected in the first few minutes, from which equatorial scattering begins to grow slowly at first $(t>4 \mathrm{~min})$ and then more rapidly later $(t>10$ min). Because we and others ${ }^{9,17}$ do not observe any discrete WAXS for $t<9 \mathrm{~min}$, the strong meridional streak must be related to a layer-shaped modulation of the electron density in an amorphous melt. The axis of the layer stack is parallel to the fiber axis. At the beginning of the equilibrium-dominated crystallization, these layers are populated by latent crystallites of first, the $c$ set, and second, the $a^{*}$ set. Weak equatorial scattering indicates lateral correlation among the crystallites. The 3D peak integrals demonstrate that meaningful weight fractions of crystallites are not available for $t<9 \mathrm{~min}$. Therefore, there is no material to build a cross-hatched topology that could be responsible for the equatorial scattering that is observed for $4<t<9$ min in the SAXS study, ${ }^{15}$ (Figure 5). Unfortunately, the meridional streak of the decomposed melt dominates the SAXS, and thus a quantitative analysis is impossible.

Only at this high crystallization temperature may the few $a^{*}$ crystallites growing from the quiescent melt be addressed as "daughter" 26 crystallites because they start later than the $c$ crystallites. Anticipating some of the results obtained for lower crystallization temperatures, our notion of the shallow-quench crystallization is sketched in Figure 15.

4.9.2. Crystallization at $145^{\circ} \mathrm{C}$. In the SAXS study ${ }^{15}$ the analysis of the patterns recorded during the first $3 \mathrm{~min}$ had only detected row assemblies ${ }^{19,26}$ of nuclei. In this period, the WAXS shows a completely amorphous material. In the interval $3<t<$ $4.5 \mathrm{~min}$, the WAXS exhibits the equilibrium-dominated regime of the crystallization. Here the SAXS, ${ }^{15}$ (Figure 9) detects a stable block structure of midrange correlation in both fiber and lateral directions. For $t>4.5 \mathrm{~min}$, the WAXS peak-integral curves, $S(t)$ and $S^{*}(t)$, indicate the growth-dominated regime of the crystallization. During this period, the evolution of the SAXS has been interpreted by a merging of the blocks into lamellae. Taking into account the evolution of $S(t)$ and $S^{*}(t)$, a different interpretation would also yield the same evolution of the SAXS. For this interpretation, it is sufficient to assume that two sets of differently oriented (block-shaped) nuclei are populating the melt and start to grow into lamellae. As the faster $c$ lamellae overtake the earlier but slower $a^{*}$ lamellae, the evolution of the SAXS suggests a merging of a $c$-set block structure into lamellae. This is a classical growth-from-nuclei mechanism that generates two differently oriented sets of layershaped domains that do not take notice of each other.

4.9.3. Crystallization at $140^{\circ} \mathrm{C}$. In the SAXS study, ${ }^{15}$ a clear evolution of domain structure has only been observed during the period up to $t=3 \mathrm{~min}$. According to the WAXS, here the growth-dominated crystallization is starting. A detailed description of the nanostructure evolution has been impossible because the time resolution has been insufficient for the fast process. Therefore, the period before the start of the nucleation (equilibrium-dominated crystallization) has not been resolved by the SAXS. The SAXS recorded during the regime of equilibriumdominated crystallization has shown a 3D arrangement ${ }^{15}$ (Figure 7) of blocks (i.e., nuclei) that turn from an entwined arrangement into a checkered arrangement, while at the same time, the gaps between the blocks shrink in both lateral and longitudinal directions. Because the WAXS shows that there is not much increase in crystallinity, the SAXS results could be explained by the notion that during the equilibrium-dominated crystallization, the amplitude of the size fluctuation of the nuclei appears to increase. The following growth-dominated phase has shown a constant SAXS pattern with increasing discrete intensity 
that has been interpreted as a dynamic equilibrium in which crystallites are emerging and ripening.

4.9.4. Crystallization Temperature and Crystallization Mechanisms. In summary, the combined results of SAXS and WAXS show that the dominance of the involved crystallization mechanisms can be adjusted by variation of the crystallization temperature.

Only upon shallow quench is a decomposition of the melt into oriented layers of different electron density clearly the first mechanism. The first nuclei observed in the structured melt belong to the $c$-oriented set. Nuclei of the $a^{*}$ set appear somewhat later. Growth of stable crystallites starts considerably later.

At medium quench depth, decomposition is not observed. Only row assemblies ${ }^{19,26}$ are found ${ }^{15}$ in the melt. After transition into the nucleation phase, the SAXS shows an arrangement of blocky crystallites. During the growth-dominated phase, the SAXS evolution can be described by merging of blocks or by a bimodal structure of differently oriented blocks that are growing and forming lamellae.

Deep quench causes very early nucleation. Again, the $a^{*}$ set is nucleated first. As the growth phase is starting, the structure is replicated all over the volume, and a superposition of structures in different states after induction is observed. Because of this dynamic equilibrium, it is impossible to separate late crystallization mechanisms.

4.10. Melting Mechanisms. In our SAXS study, ${ }^{15}$ we had already detected a sequence of several melting mechanisms in the industrial HEPP. In the temperature range up to $158^{\circ} \mathrm{C}$, we had observed the melting of secondary lamellae that are less extended in the equatorial direction. Now, the WAXS data (Figures 6 and 10) show that these domains contain the $a^{*}$ oriented crystallites. This result is in agreement with several studies ${ }^{3,5,26,47}$ in which $a^{*}$-oriented crystallites generated under extensional flow conditions were found to be of secondary nature.

For $158<T<162{ }^{\circ} \mathrm{C}$, the SAXS study had exhibited a considerable decrease in the average lateral extension of the lamellae, and an increase in the average long period had been found. This mechanism had been interpreted as the melting of extended secondary lamellae. In this temperature interval, the WAXS data show that the weight crystallinity of perfect crystallites with normal chain orientation $(S(t, T))$ is only subject to little change (Figures 6 and 10). This finding can be explained by recrystallization, that is, the melting of crystallites inside imperfect lamellae that is superimposed by crystallization of distorted zones inside these secondary lamellae.

For $T>162{ }^{\circ} \mathrm{C}$, the SAXS study ${ }^{15}$ showed a third melting mechanism: "The long period remains almost constant and the average layer extension is increasing, indicating the melting of (now the primary) crystalline lamellae with the less extended layers melting first". Also, the WAXS data exhibit a third mechanism. At $162{ }^{\circ} \mathrm{C}$, the weight crystallinity of the $c$ set abruptly falls off. Thereafter, it decreases more slowly following a sigmoidal course until it reaches the value zero. This finding is in agreement with the explanation given in the SAXS study. The weight of the crystallites inside the secondary lamellae abruptly decreases as soon as they are no longer nourished from the distorted zones. Thereafter, the primary lamellae melt from edge to core, and the sigmoidal decay is caused by the size distribution of the primary lamellae. Therefore, these primary lamellae of the $c$ set appear to be grown from nuclei.

4.11. Second Melting. As was done in our SAXS study, ${ }^{15}$ we have molten the samples after the crystallization experiment for a second time. Figure 16 shows the result for the sample that had been crystallized at $145^{\circ} \mathrm{C}$ before. After the crystallization experiment, the minimum lateral extension, $b^{*}$, of crystallites in $a^{*}$ orientation is much larger than that in the industrial HEPP (cf. Figures 6 and 10). Here the $a^{*}$-set crystallites show a melting behavior that is very similar to that of the $c$-set crystallites. The apparently somewhat earlier melting $\left(t_{0 \mathrm{~m}}<t_{0 \mathrm{~m}}^{*}\right.$ with $\left.S\left(t_{0 \mathrm{~m}}\right)=0, S^{*}\left(t_{0 \mathrm{~m}}^{*}\right)=0\right)$ of the $c$-set crystallites is not considered to be significant. The $c$-set 110 reflection is much broader than the quasi-meridional reflection of the recrystallized $a^{*}$ set. Close to its disappearance, it is more difficult for the peak-extraction procedure to separate the $c$-set reflection from the noise than it is for the sharp $a^{*}$-set reflection. In the case of the anticipated systematic deviation, the falloff of the determined curve $S(t)$ should considerably increase before it vanishes because the tails of the reflection drown in the noise. The difference between the extrapolation line shown in Figure 16 and the measured curve is indicative for such an effect. In summary, the material recrystallized from the quiescent melt exhibits a simple melting mechanism that is very similar for both of the differently oriented crystallite sets.

\section{Conclusions}

Variation of process parameters causes considerable variation of the crystallization mechanisms and the resulting nanostructure of PP. There is no simple general crystallization mechanism. This is not only demonstrated by the differences among the materials monitored at different crystallization temperature but, in particular, after comparing these results to the structure and the melting mechanisms found in the commercial starting material (HEPP). Obviously, evolution mechanisms observed under isothermal conditions in a quiescent melt cannot be simply transferred to the behavior of polymer melts crystallizing under gradients of temperature and pressure that are typical of industrial processes.

It is generally reported that the $c$-set crystallites are formed during primary crystallization, whereas the $a^{*}$-oriented crystallites are of secondary nature ("daughter lamellae", "crosshatched lamellae"). 3,26,47 Our results demonstrate that this may not be the case for a crystallization from an oriented quiescent melt. Upon deep quench, crystallization starts in the $a^{*}$ set. In contrast, the industrial process in which the HEPP material is produced is obviously governed by the commonly reported sequence. In the commercial material, the reflections of the $a^{*}$ set crystallites are much broader than those after recrystallization, and the small $a^{*}$-set crystallites of HEPP melt at a much lower temperature than the $c$-set crystallites.

Application of the new automatic method for the computation of 3D peak integrals in reciprocal space has resulted in plots demonstrating that crystallite formation is a two-stage process. It is observed in both the homogeneous and the decomposed melts (i.e., deep and shallow quench). In the first stage, there is little increase in the very low crystallinity until crystallinity starts to grow rapidly in the second stage. Therefore, the historical discussion concerning the sensitivity ${ }^{9,11-13}$ of WAXS detectors appears to be settled for oriented samples: Even the available standard detectors already register WAXS reflections in the first stage, in which the crystallinity remains so low that it does not yet contribute to the SAXS. In our experiments, we have not observed rigid stable shish-kebab nuclei that have recently been observed in the group of Hsiao ${ }^{48}$ with polyethylene and that should not melt ${ }^{49}$ under the mild melting conditions chosen by us. After the combination of the older SAXS results with the new WAXS data, the shallow-quench meridional streak in the SAXS appears to be no longer related to crystalline lamellae but to a decomposed melt. The same has been found with polyethylene. ${ }^{14,50}$ In particular, it looks as if the layer-arrangement mechanism of "random car parking" "51-53 is not characterizing crystallization itself but a precedent decomposition of the melt. A possible explanation for the fact that decomposition is 
not detected in some studies of isotropic PP crystallization may be related to a combination of chemical composition, sample history, quench depth, solid-angle average of the SAXS, background subtraction, and definition of the zero time.

Admittedly, the combination of results from successively executed SAXS and WAXS experiments is of limited significance as long as the exact temperature in the irradiated volume of the sample cannot be measured. Simultaneous measurements of anisotropic scattering that provide complete patterns for quantitative analysis will not be possible until detectors with a central hole and electronic read-out become available. A detector device that is assembled from tiles ${ }^{54}$ might be adaptable by the omission of a central tile.

Acknowledgment. We thank J. C. Gregor for carrying out the 1D WAXS trend analysis and the Hamburg Synchrotron Radiation Laboratory (HASYLAB) for beam time granted in the frame of project II-04-039. We gratefully acknowledge funding by the Deutsche Forschungsgemeinschaft, project STR 501/4-2. Part of this work has been supported by the seventh framework program of the European Union (project NANOTOUGH FP7-NMP-2007LARGE).

\section{References and Notes}

(1) Compostella, M.; Coen, A.; Bertinotti, F. Angew. Chem. 1962, 74, 618.

(2) Clark, E. S.; Spruiell, J. E. Polym. Eng. Sci. 1976, 16, 176.

(3) Katayama, K.; Amano, T.; Nakamura, K. Kolloid Z. Z. Polym. 1968, $226,125$.

(4) Norton, D. R.; Keller, A. Polymer 1985, 26, 704.

(5) Olley, R. H.; Bassett, D. C. Polymer 1989, 30, 399.

(6) Li, H.; Sun, X.; Wang, J.; Yan, S.; Schultz, J. M. J. Polym. Sci., Part B: Polym. Phys. 2006, 44, 1114.

(7) Albrecht, T.; Strobl, G. Macromolecules 1995, 28, 5267.

(8) Ryan, A. J.; Terrill, N. J.; Fairclough, J. P. A. ACS Symp. Ser. 2000, 739, 201.

(9) Heeley, E. L.; Maidens, A. V.; Olmsted, P. D.; Bras, W.; Dolbnya, I. P.; Fairclough, J. P. A.; Terrill, N. J.; Ryan, A. J. Macromolecules 2003, 36, 3656.

(10) Dai, P. S.; Cebe, P.; Capel, M.; Alamo, R. G.; Mandelkern, L. J. Appl. Crystallogr. 2000, 33, 714.

(11) Wang, Z.-G.; Hsiao, B. S.; Sirota, E. B.; Agarwal, P.; Srinivas, S. Macromolecules 2000, 33, 978.

(12) Panine, P.; Urban, V.; Bösecke, P.; Narayanan, T. J. Appl. Crystallogr. 2003, 36, 991.

(13) Panine, P.; Di Cola, E.; Sztucki, M.; Narayanan, T. Polymer 2008, $49,676$.

(14) Stribeck, N.; Almendarez Camarillo, A.; Cunis, S.; Bayer, R. K.; Gehrke, R. Macromol. Chem. Phys. 2004, 205, 1445.

(15) Stribeck, N.; Nöchel, U.; Almendárez Camarillo, A.; Roth, S. V.; Dommach, M.; Bösecke, P. Macromolecules 2007, 40, 4535.

(16) Somani, R. H.; Hsiao, B. S.; Nogales, A.; Srinivas, S.; Tsou, A. H.; Sics, I.; Baltá Calleja, F. J.; Ezquerra, T. A. Macromolecules 2000, 33,9385 .
(17) Somani, R. H.; Yang, L.; Hsiao, B. S.; Agarwal, P. K.; Fruitwala, H. A.; Tsou, A. H. Macromolecules 2002, 35, 9096.

(18) Somani, R. H.; Yang, L.; Hsiao, B. H.; Fruitwala, H. J. Macromol. Sci., Phys. 2003, B42, 515.

(19) Nogales, A.; Mitchell, G. R. Polymer 2005, 46, 5615.

(20) Ogino, Y.; Fukushima, H.; Takahashi, N.; Matsuba, G.; Nishida, K.; Kanaya, T. Macromolecules 2006, 39, 7617.

(21) Elias, H.-G. Polymere: Von Monomeren und Makromolekülen zu Werkstoffen; Hüthig und Wepf: Heidelberg, Germany, 1996.

(22) Suaphol, P.; Lin, J.-S. Polymer 2001, 42, 9617.

(23) Cho, K.; Saheb, D. N.; Choi, J.; Yang, H. Polymer 2003, 43, 1407.

(24) De Rosa, C.; Ruiz de Ballesteros, O.; Di Gennaro, M.; Auriemma, F. Polymer 2003, 44, 1861.

(25) Xu, J.; Srinivas, S.; Marand, H.; Agarwal, P. Macromolecules 1998, 31,8230 .

(26) Kumaraswamy, G.; Verma, R. K.; Issaian, A. M.; Wang, P.; Kornfield, J. A.; Yeh, F.; Hsiao, B. S.; Olley, R. H. Polymer 2000, 41, 8931.

(27) Hugel, T.; Strobl, G.; Thomann, R. Acta Polym. 1999, 50, 214.

(28) Strobl, G. Prog. Polym. Sci. 2006, 31, 398.

(29) Stribeck, N. Acta Crystallogr. 2009, A65, 46.

(30) Stribeck, N.; Nöchel, U. J. Appl. Crystallogr. 2009, in press.

(31) Noether, H. D. Int. J. Polym. Mater. 1979, 7, 57.

(32) Sprague, B. S. J. Macromol. Sci., Phys. 1973, B8, 157.

(33) Noether, H. D.; Whitney, W. Colloid Polym. Sci. 1973, 251, 991.

(34) Mencik, Z. J. Macromol. Sci., Phys. 1972, B6, 101.

(35) Polanyi, M. Z. Phys. 1921, 7, 149.

(36) Stribeck, N. X-Ray Scattering of Soft Matter; Springer: New York, 2007.

(37) Fraser, R. D.; Macrae, T. P.; Miller, A.; Rowlands, R. J. J. Appl. Crystallogr. 1976, 9, 81

(38) Millane, R. P.; Arnott, S. J. Macromol. Sci., Phys. 1985, B24, 193.

(39) Stribeck, N. Polymer Physics Downloads.http://www.chemie.unihamburg.de/tmc/stribeck/dl ( 2008).

(40) Buhmann, M. D. Acta Numerica 2000, 9, 1.

(41) Haberäcker, P. Digitale Bildverarbeitung; Hanser: Munich, Germany, 1989.

(42) PV-WAVE manuals, version 7.5; VNI: Boulder, CO, 2007.

(43) Ruland, W. Acta Crystallogr. 1961, 14, 1180.

(44) Alexander, L. E. X-Ray Diffraction Methods in Polymer Science; Wiley: New York, 1979.

(45) Warren, B. E. X-Ray Diffraction; Dover: New York, 1990.

(46) Guinier, A. X-Ray Diffraction; Freeman: San Francisco, 1963.

(47) Sakthivel, A.; Abhiraman, S. J. Appl. Polym. Sci. 1984, 29, 4257.

(48) Keum, J. K.; Zuo, F.; Hsiao, B. S. Macromolecules 2008, 41, 4766.

(49) Janeschitz-Kriegl, H.; Eder, G. J. Macromol. Sci., Phys. 2007, 46, 591.

(50) Stribeck, N.; Almendarez Camarillo, A.; Nöchel, U.; Bösecke, P.; Bayer, R. K. Anal. Bioanal. Chem 2007, 387, 649.

(51) Rényi, A. Sel. Transl. Math. Stat. Prob. 1963, 4, 203.

(52) Schultz, J. M.; Lin, J. S.; Hendricks, R. W. J. Appl. Crystallogr. 1978, $11,551$.

(53) Stribeck, N. Macromol. Chem. Phys. 2004, 205, 1455.

(54) Broennimann, C.; Eikenberry, E. F.; Henrich, B.; Horrisberger, R.; Hülsen, G.; Pohl, E.; Schmitt, B.; Schulze-Briese, C.; Suzuki, M.; Tomizaki, T.; Toyokawa, A.; Wagner, A. J. Synchrotron Rad. 2006, $13,120$.

MA8025282 